five years ago, the consumer of gas for power or heating purposes has now to burn about 1200 cubic feet of gas in the place of rooo, costing $3^{s} .2 \frac{1}{2} d$. as against $3 s$., plus a meter rental (varying with the consumption), plus the extra cost of repairs caused by the additional sulphur present. The consumer, for lighting purposes, if using throughout an incandescent mantle, is not seriously prejudiced; if, however, he retains the batswing. burner, his outlay for the same amount of light has increased in the ratio of about $4 / 3$, plus a meter rental and plus an increased cost of interial decoration due to the condensation on the walls and ceilings of an increased amount of sulphuric acid.

THE Scientific American for January ${ }_{5} 5$ shows an illustration of the McClean-Lissack automatic rapid-fire gun, which was tested last year by the Ordnance Department of the United States Army. This gun is designed for attacking balloons, and is mounted on a Packard 3 -ton automobile truck. The gun fired $3^{-1 b}$. shots at the rate of 100 per minute, the range being $3 \frac{1}{2}$ miles. With brakes on, the truck did not move on firing, and no shock was perceived by those standing on the truck platform. With brakes released there was a slight movement on the recoil, but no shock: Further tests with this gun are being made at Sandy Hook and Springfield for the army, and at Indian Head for the navy. The same article also illustrates two German automobile guns designed for the same purpose. One of these is mounted on an armoured truck of 60 horsepower, capable of a speed of 45 kilometres per hour. The shell from this gun has a maximum height of trajectory of 3800 metres.

THE ninth report to the alloys research committee was presented by Dr. W. Rosenhain and Mr. F. C. A. H. Lantsberry at the meeting of the Institution of Mechanical Engineers:on Friday, January 21. Dr. Rosenhain explained that this report dealt with the properties of some alloys of copper, aluminium; and manganese, and is confined to some of the more interesting alloys likely to be of practical service. The greater part of the work was confined to alloys containing less than if fer cent. of aluminium, and also less than in per cent. of manganese. It is impossible to state adequately and briefly the enormous amount of valuable information resulting from this research -the report occupies $x 74$ pages of the institution's transactions. Specific mention might be made of the great tensile strength exhibited by one of the alloys in the form of a cold-drawn bar, having a yield point of 40.88 tons per square inch and an ultimate stress of $5^{2.08}$ tons per square inch. This alloy had 9.99 per cent. aluminium, 2.01 per cent. manganese, and 88 per cent. copper. Another alloy shows hardness sufficient to enable it to take a cutting edge that will sharpen a lead pencil. In addition to the mechanical tests and microscopic and freezing-point investigations, corrosion in sea water has been examined. Further and more searching tests on the latter are now proceeding at Portsmouth Harbour, and have also been arranged for in the warmer sea water at Malta Dockyard.

The January number, of the Journal of the Royal Statistical Society begins a new series of the journal, to be issued monthly during the session. It is hoped that the greater rapidity of publication thus secured will be of service, as papers read one month will now be in the hands of fellows, and the public generally, by the middle of the following month instead of sometimes not appearing for three months or more, as is necessarily the case with a quarterly journal. Current notes also form a new section of the journal which it is hoped will increase its general interest.

NO. 2 IOI, VOL. 82]
OUR ASTRONOMICAL COLUMN.

Astronomical. OCCURRENCES In FEBruary:-

Feb. 4. 5h. Venus in pesihelion.

6. I2h. Mercury stationary.

7. I2h. $25 \mathrm{~m}$. Uranus in conjunction with the Moon (Uranus $3^{\circ} 19^{\prime} \mathrm{N}$.).

9. $12 \mathrm{~h} .36 \mathrm{~m}$. Venus in conjunction with the Moon (Venus $13^{\circ} 34^{\prime} \mathrm{N}$.)

12. Oh. Venu, in in'erior conjunction with the Sun.

I3. I3h. $22 \mathrm{~m}$. Saturn in conjunction with the Moon (Saturn $I^{\circ}$ I $8^{\prime}$ N.).

15. 6h. 12m. Mars in conjunction with the Moon $\left(\operatorname{Mars} 3^{\circ} \mathbf{I}^{\prime}\right.$ N.).

I9. I $7 \mathrm{~h}$. Mercury at greatest elongation west of the Sun:

I9. 2 Ih. $33 \mathrm{~m}$. Neptune in conjunction with the Moon (Neptune $4^{\circ}$ Io' S.).

26. $3^{\text {h. }}$. Venus at greatest hel:ocentric latitude north.

, $17 \mathrm{~h}$. $34 \mathrm{~m}$. Jupiter in conjuncion with the Moon (Jupiter $2^{\circ} 29^{\prime}$ S.).

Mars.-Readers of these columns should be fairly well acquainted with Prof. Lowell's views concerning the Martian features and their significance, but they will find interesting the comprehensive summary given by Prof Lowell in No. ${ }^{3} 3$ of Scientia, the international science review published at Bologna, and obtainable from Messrs. Williams and Norgate. Therein the author reviews the observations of the melting snow-caps, of the "canals" and oases, which, by virtue of their dependent vegetation, undergo striking changes in conformity with the Martian seasons, and the theoretical considerations which have led him to conclude that Mars is habitable by organisms not essentially different from those with which we are acquainted. That Mars has no water except that contained in its atmosphere and that which forms the snow-caps, Prof: Lowell avers, but he contends that that water is artificially " engineered". in such a way that organic existence is rendered possible.

Ciroline Herschel and Her Comet Seeker. - At the present moment, with the subject of comets so much to the fore, an article which appears in the January number of Himmel und Erde is of especial interest. The writer gives many details of Caroline Herschel's strenuous life and describes her labours with the comet seeker. A facsimile reproduction of a letter, dated August 5, I $83 \mathrm{I}$, from her to Director Hausmann, tells how the comet seeker was made and how she wished it to be used after she had finished with it. The instrument was made of odds and ends by her brother " between breakfast and dinner." "The tube had once been used as a Newtonian finder to the 2o-foot reflector. The circular board once served for a fly-wheel in some experiment; and for the pole, I was sent to the scullery to find a mopstic. The rest was sawed and chopped in the shapes as they were wanted-as for plaining we could do without, there was no time for niceties." Yet, she adds, it stood for fortyseven years without wanting a single repair, travelling all over the house and garden, at Slough, many a night; and with it she discovered five of the eight comets credited to her name.

\section{EDDY FORMATION IN THE WAKE OF PROJECTING OBSTACLES.}

CONSIDER a stream bounded by and moving parallel to the plane $\mathrm{OX}$, with velocity $\mathrm{U}$, and containing a stationary vortex at $\mathrm{A}(a, b)$, or, what is the same thing, an unbounded fluid containing a stationary vortex-pair at A, B (Fig. I). With the notation $w=\phi+\imath \psi, z=x+\imath y$, $\phi=$ velocity potential, $\psi=$ stream function, the potential function is given, for this case, by

$$
w=\mathrm{U}\left(z+2 i b \log \frac{z-a-i b}{z-a+i b}\right) .
$$

Inside a certain surface $O Q P$, the stream lines are closed curves and the motion is cyclic; outside, the fluid streams past the surface as if it were a solid obstacle, as is well 